Check for updates

Cite this: RSC Adv., 2018, 8, 1255

Received 25th October 2017

Accepted 22nd December 2017

DOI: 10.1039/c7ra11770a

rsc.li/rsc-advances

\section{Chitosan-derived three-dimensional porous carbon for fast removal of methylene blue from wastewater}

\author{
Qian Jin, (D) ${ }^{a}$ Yadong Li, (iD) ${ }^{a}$ Desong Yang ${ }^{\star a}$ and Jianghu Cui (DD *b
}

Despite much progress in modifying chitosan as an absorbent for wastewater treatment, it is still difficult for current chitosan-based adsorbents to achieve the desired removal effects towards basic dyes. In this study, chitosan-derived three-dimensional porous carbon (CTC) consisting of large-diameter channels and mesopores was prepared to remove methylene blue (MB) from wastewater. The results indicate that CTC has excellent performance for MB removal, and the maximum adsorption capacity was $925.93 \mathrm{mg} \mathrm{g}^{-1}$ at $318 \mathrm{~K}$. The adsorption isotherm and kinetics models of MB on CTC could be described well by the Langmuir isotherms and the pseudo-second-order rate model. An experiment to study the CTC removal of MB from a flowing aqueous solution was performed using a homemade device. The water treatment rate of CTC reached $250 \mathrm{~L} \mathrm{~g}^{-1} \mathrm{~h}^{-1}$, with high MB removal efficiency (>93.4\%). Furthermore, the desorption-adsorption experiments indicate that CTC is also a reusable adsorbent that can be applied to recover MB from wastewater. The obtained CTC is a promising alternative for the current expensive absorbents and provides a concept for designing the three-dimensional (3D) structures of raw materials to improve adsorption capability.

\section{Introduction}

Dyes are used today more than ever in industries such as textiles, paper, printing, leather tanning, food processing, rubber, pulp mills, cosmetics and plastics. ${ }^{\mathbf{1} 2}$ The worldwide consumption of dyes has exceeded 700000 tons annually in recent years, and approximately $10-20 \%$ is discharged into water. ${ }^{3}$ Given their varying stability to light, oxidants and microorganisms, dyes are degraded at varying rates. ${ }^{4}$ In addition, dyes are toxic and even carcinogenic to humans when they are released into the aquatic environment. ${ }^{5,6}$ Therefore, it is necessary to remove dyes from wastewater before they are released into the environment. For the effective removal of dyes from wastewater, several methods including physical, chemical and biological methods have been investigated. ${ }^{7-9}$ Among the proposed methods, adsorption technologies are regarded as a competitive method for high efficiency, economic feasibility and simplicity of design/operation. ${ }^{\mathbf{1 0}}$

Some adsorbents have been investigated in detail, including activated carbon, ${ }^{11,12}$ cellulose ${ }^{13}$ inorganic oxides, ${ }^{14}$ anaerobic sludge $^{15}$ and chitosan. ${ }^{16}$ Among these adsorbents, activated carbon is the most common adsorbent for the efficient removal

${ }^{a}$ College of Agriculture, Shihezi University, Shihezi 832000, Xinjiang, P. R. China. E-mail: yds_agr@shzu.edu.cn

${ }^{b}$ Guangdong Key Laboratory of Integrated Agro-environmental Pollution Control and Management, Guangdong Institute of Eco-environmental Science \& Technology, Guangzhou 510650, China. E-mail: Lantian9426@163.com of dyes from wastewater. However, activated carbon is usually prepared from relatively expensive materials, limiting its wide application. Some alternative low-cost adsorbents have received attention, such as the biopolymer chitin and its derivative chitosan. ${ }^{\mathbf{1 7 , 1 8}}$ Chitin is a common primary material and widely distributed in the exoskeletons of crustaceans or fungal cell walls. ${ }^{19}$ Chitosan is a natural cationic polymer that can be obtained by $\mathrm{N}$-deacetylation of chitin. ${ }^{\mathbf{1 7 , 2 0}}$ In terms of adsorption capacity, chitosan shows more excellent adsorption performance than chitin, mainly due to its abundant hydroxyl functional groups and free amino $\left(-\mathrm{NH}_{2}\right)$ groups. Chitosan is an excellent adsorbent for anionic and reactive dyes due to its chemical structure. ${ }^{21-23}$ However, chitosan shows weak adsorption performance for basic dyes. ${ }^{24,25}$ Due to low surface area and instability, ${ }^{26}$ chitosan is not an ideal absorbent for fast wastewater treatment. Thus, it is promising to modify chitosan to improve its adsorption capability for basic dyes and stability in various wastewaters.

To date, much modification research has focused on improving the adsorption capacity of chitosan or widening the range of application for various dyes, such as quarterized chitosan, ${ }^{25}$ magnetic chitosan, ${ }^{27,28}$ chitosan-activated carbon, ${ }^{29}$ chitosan clay, ${ }^{30}$ polyvinyl alcohol chitosan, ${ }^{31}$ and cross-linked chitosan. $^{32}$ However, most modifications only changed the chitosan-active groups, not its original structures. ${ }^{33}$ Recently, through the design of $3 \mathrm{D}$ porous structures in some natural materials, excellent properties have been found, including catalysis, adsorption and electrochemical activities. ${ }^{34-36}$ The 3D 
porous structures not only reduce the original density of the material but also improve the exposure and accessibility of active sites to the target, ${ }^{37,38}$ which should be very useful for adsorption. The reported adsorbents generally had good adsorption capability at equilibrium, but the time interval of the equilibrium condition is long for most adsorbents, especially for chitosan-based adsorbents. ${ }^{39-41}$ To date, only a few 3D porous materials have been reported to be applied for the fast removal of dye from wastewater. ${ }^{42}$ Therefore, it is meaningful to introduce $3 \mathrm{D}$ porous structures into the adsorbent for the fast removal of dye from wastewater.

Here, we used chitosan as a carbon precursor to prepare a 3D porous structures adsorbent, which we named CTC. The main processes included pre-carbonization and activation with $\mathrm{KOH}$ at various temperatures. To study the adsorption capability of CTC towards basic dyes, MB was used as a representative basic dye. A homemade device was made to simulate rapid removal of basic dyes from flowing water. The results indicated that CTC not only showed a high adsorption capability towards $\mathrm{MB}$ at equilibrium but could also be applied for the fast removal of $\mathrm{MB}$ from flowing wastewater. The whole process of adsorption is feasible, spontaneous, and exothermic, and no secondary waste was produced. Furthermore, the obtained CTC is reusable for the removal of $\mathrm{MB}$ from wastewater. In summary, CTC is a promising adsorbent for real large-scale application.

\section{Experimental section}

\subsection{Materials}

Chitosan was purchased from the Guangzhou Qiyun Biotechnology Co. Ltd. According to the manufacturer's instructions, its molecular weight is $450 \mathrm{kDa}$, and the degree of deacetylation is above 95\%. $\mathrm{KOH}$ was obtained from the Tianjin Fuchen Chemical Reagent Factory. During this experiment, the applied reactants were of analytical grade. MB (chemical formula: $\mathrm{C}_{16} \mathrm{H}_{18} \mathrm{~N}_{3} \mathrm{SCl} \cdot 3 \mathrm{H}_{2} \mathrm{O}$; molecular weight: 373.9 ; $\lambda_{\max }=$ $665 \mathrm{~nm}$ ) was a basic dye purchased from the Tianjin Damao Chemical Reagent Factory. Ethylene Diamine Tetraacetic Acid (EDTA) was purchased from Sigma. Oxalic acid was purchased from the Shanghai Macklin Biochemical Technology Company. In this study, the purity of the dyes was the original purity, without further purification.

\subsection{Synthesis of CTC}

Chitosan was directly heated to $450{ }^{\circ} \mathrm{C}$ in the presence of nitrogen at a heating rate of $5^{\circ} \mathrm{C} \min ^{-1}$ and kept for $4 \mathrm{~h}$ at this temperature. The pre-carbonized char was mixed with $\mathrm{KOH}$, and the impregnation ratio of $\mathrm{KOH} / \mathrm{char}$ was 4 . The mixtures were heated to $450{ }^{\circ} \mathrm{C}$ with a heating rate of $5{ }^{\circ} \mathrm{C} \mathrm{min}{ }^{-1}$ and maintained for $0.5 \mathrm{~h}$ at this temperature, followed by heating from $450{ }^{\circ} \mathrm{C}$ to $600{ }^{\circ} \mathrm{C}$ with a heating rate of $5{ }^{\circ} \mathrm{C} \mathrm{min}{ }^{-1}$ and holding for $0.5 \mathrm{~h}$ at $600{ }^{\circ} \mathrm{C}$, with a final heating from $600{ }^{\circ} \mathrm{C}$ to $900{ }^{\circ} \mathrm{C}$ at a heating rate of $5{ }^{\circ} \mathrm{C} \mathrm{min}^{-1}$, with a constant final temperature held for $1.5 \mathrm{~h}$. After activation, the resulting CTC was ground, washed with an $0.1 \mathrm{M} \mathrm{HCl}$ solution and then distilled water until the $\mathrm{pH}$ was approximately 7 , and it was dried at $105{ }^{\circ} \mathrm{C}$ overnight. The obtained sample was stored in a desiccator for later use. The quality of the output after each heating phase was counted. According to the counted data, the final yield of the CTC material was $22.15 \%$.

\subsection{Characterisation of CTC}

The XRD patterns for chitosan and CTC were obtained with a Philips X'pert diffractometer using $\mathrm{Cu} \mathrm{K} \alpha$ radiation at $36 \mathrm{kV}$ and $20 \mathrm{~mA}$ over the $2 \theta$ range of $10-80^{\circ}$. The FTIR spectra for chitosan and CTC were recorded on a Nicolet Nexus 470 FTIR spectrometer in the range of $400-4000 \mathrm{~cm}^{-1}$ using $\mathrm{KBr}$ tablets containing the prepared materials. CTC structures were observed by SEM. The values of the BET surface areas of CTC and chitosan were obtained from the adsorption of $\mathrm{N}_{2}$ at $77 \mathrm{~K}$ using an accelerated surface area and porosimetry system (Tristar 3000, Micromeritics) and were calculated from the $\mathrm{N}_{2}$ adsorption/desorption isotherm data.

\subsection{Adsorption experiments}

The adsorption experiments for MB were conducted by adding various doses of adsorbent into $100 \mathrm{~mL}$ of $\mathrm{MB}$ solutions with different initial concentrations. During adsorption, a thermostatted agitator was set at $140 \mathrm{rpm}$ to perform the batch experiments. The absorbance values of residual dye solution were obtained by UV-vis spectrophotometry (Cary 50, Varian, USA) at a wavelength of $665 \mathrm{~nm}$ after filtration from the adsorbents. The flow rate is controlled by a vacuum pump (GM0.33A, JINTENG, China), and the pH of the solution was adjusted with $\mathrm{HCl}(0.1 \mathrm{M}$ and $0.01 \mathrm{M})$ and $\mathrm{KOH}(0.1 \mathrm{M}$ and 0.01 $\mathrm{M})$ in the fast wastewater treatment experiments. We obtained accurate values for residual MB concentrations using the linear regression of absorbance to the calibration curve of $\mathrm{MB}$. The following equations were used to calculate the removal efficiency $(R \%)$ and the amount of adsorbed $\mathrm{MB}$ at time $t$ $\left(q_{t}, \mathrm{mg} \mathrm{g}^{-1}\right)$ and at equilibrium $\left(q_{\mathrm{e}}, \mathrm{mg} \mathrm{g}^{-1}\right)$.

$$
\begin{aligned}
& R=100 \times \frac{C_{0}-C_{\mathrm{e}}}{C_{0}} \\
& q_{t}=\frac{\left(C_{0}-C_{t}\right) \times V}{m} \\
& q_{\mathrm{e}}=\frac{\left(C_{0}-C_{\mathrm{e}}\right) \times V}{m}
\end{aligned}
$$

where $C_{0}, C_{t}$ and $C_{\mathrm{e}}\left(\mathrm{mg} \mathrm{\textrm {L } ^ { - 1 }}\right)$ are the concentrations of $\mathrm{MB}$ solution at initial, time $t$ and equilibrium, respectively. $V(\mathrm{~L})$ stands for the volume of MB solution, and $m(\mathrm{~g})$ is the mass of the adsorbent (CTC).

\subsection{Desorption experiments}

Desorption studies were performed by mixing $0.1 \mathrm{~g}$ of adsorbents with $50 \mathrm{~mL}$ of an aqueous $\mathrm{MB}$ solution at $100 \mathrm{mg} \mathrm{L}^{-1}$. The mixture was shaken at $140 \mathrm{rpm}$ and $30{ }^{\circ} \mathrm{C}$ for $8 \mathrm{~h}$. The solid phase was collected by filtration and washed several times with distilled water to remove any unabsorbed MB. MB-loaded adsorbents were regenerated in $50 \mathrm{~mL}$ of desorbing agents 
(distilled water, 0.1 M of EDTA, 0.1 M of hydrochloric acid, ethyl alcohol, and $0.1 \mathrm{M}$ of oxalic acid) on a rotary shaker at $140 \mathrm{rpm}$ for $8 \mathrm{~h}$. The concentrations of $\mathrm{MB}$ in the elutes were determined to investigate the desorption efficiency, and the material was washed with distilled water until a neutral $\mathrm{pH}$ was obtained. The regenerated adsorbent was dried at $60^{\circ} \mathrm{C}$ and reused in the next cycle of adsorption experiments.

\section{Results and discussion}

\subsection{Synthesis and characterization of CTC}

In this study, we used chitosan as a precursor. A simple synthesis comprised pre-carbonization and activation with $\mathrm{KOH}$ at different temperatures. Typical XRD patterns of CTC and chitosan are shown in Fig. 1a. The chitosan has a broad peak at $2 \theta=20^{\circ}$, which is a crystalline feature of chitosan. ${ }^{43}$ After pre-carbonization and activation, the characteristic peak disappeared in the XRD pattern of CTC. This change was considered for the effect of high carbonization temperature and the activation of $\mathrm{KOH}$, which might destroy the crystalline structure in the chitosan completely. Therefore, CTC contains mainly amorphous components. The nitrogen adsorption/ desorption isotherms of chitosan and CTC were also presented in Fig. 1b. The isotherm of CTC corresponds to the type IV B.D.D.T classification, ${ }^{44}$ meaning that CTC is typical of mesoporous materials, while untreated chitosan can be considered as non-porous. According to the adsorption data, the BET surface areas of CTC were $3496 \mathrm{~m}^{2} \mathrm{~g}^{-1}$, while that of chitosan were only $0.70 \mathrm{~m}^{2} \mathrm{~g}^{-1}$. Thus, pre-carbonization and activation contributed a large surface area for CTC. The mechanism mainly included the reaction between $\mathrm{KOH}$ and pre- carbonized chitosan. During this process, the pores in the absorbent were constantly generated and expanded, with the depletion of carbon molecules from the char network and the generation of gas such as $\mathrm{H}_{2}, \mathrm{CO}$ and $\mathrm{CO}_{2} \cdot{ }^{45-47}$ The generated soluble potassium salt was washed away, leaving rich pores. The pore size distributions of the CTC are shown in Fig. 1c. The results indicated that these samples contain small mesopores ( 2 to $8 \mathrm{~nm}$ ). The surface functional groups of chitosan and CTC were also characterized by the FTIR spectrum. The results are shown in Fig. 1d. The principal spectral features of chitosan are as follows: $3380 \mathrm{~cm}^{-1}(\mathrm{O}-\mathrm{H}$ stretch superimposed on $\mathrm{N}-\mathrm{H}$ stretch and inter- and extra-molecular hydrogen bonds of the polysaccharide), $2918 \mathrm{~cm}^{-1}$ and $2878 \mathrm{~cm}^{-1}$ (C-H stretch), $1647 \mathrm{~cm}^{-1}$ (amide I band, $\mathrm{C}=\mathrm{O}$ stretch), $1597 \mathrm{~cm}^{-1}\left(\mathrm{NH}_{2}\right.$ bending), $1383 \mathrm{~cm}^{-1}$ (amide III band, C-N stretch), $1420 \mathrm{~cm}^{-1}$ and $1323 \mathrm{~cm}^{-1}$ (C-H bending), $1259 \mathrm{~cm}^{-1}$ (C-N stretch), $1155 \mathrm{~cm}^{-1}$ (bridge C-O-C stretch), and $1082 \mathrm{~cm}^{-1}$ and $1030 \mathrm{~cm}^{-1}$ (C-O stretch). ${ }^{48,49}$ Compared to chitosan, the FTIR spectral peak intensity of CTC at $1647 \mathrm{~cm}^{-1}$ decreased. This means that the $\mathrm{C}-\mathrm{N}$ bond of the amide was broken after precarbonization and activation with $\mathrm{KOH}$ at various temperatures. In this process, gases were generated, including $\mathrm{CO}, \mathrm{H}_{2}$ and $\mathrm{CO}_{2}$, leading to the formation of porous structures in CTC. The disappearance of the peaks at $1420 \mathrm{~cm}^{-1}$ and $1323 \mathrm{~cm}^{-1}$ for CTC can be attributed to fracturing of the $\mathrm{C}-\mathrm{H}$ bond. The peaks at $1383 \mathrm{~cm}^{-1}$ and $1259 \mathrm{~cm}^{-1}$ decreased or disappeared. This is due to the decomposition of the $\mathrm{N}-\mathrm{H}$ linkage. Meanwhile, the CTC peaks at $1155 \mathrm{~cm}^{-1}$ (bridge C-O-C stretch), $1082 \mathrm{~cm}^{-1}$ and $1030 \mathrm{~cm}^{-1}$ (C-O stretch) disappeared, possibly because both the bridge $\mathrm{C}-\mathrm{O}-\mathrm{C}$ and $\mathrm{C}-\mathrm{O}$ bond were almost completely destroyed. Hence, the FTIR spectrum of CTC demonstrates that the surface
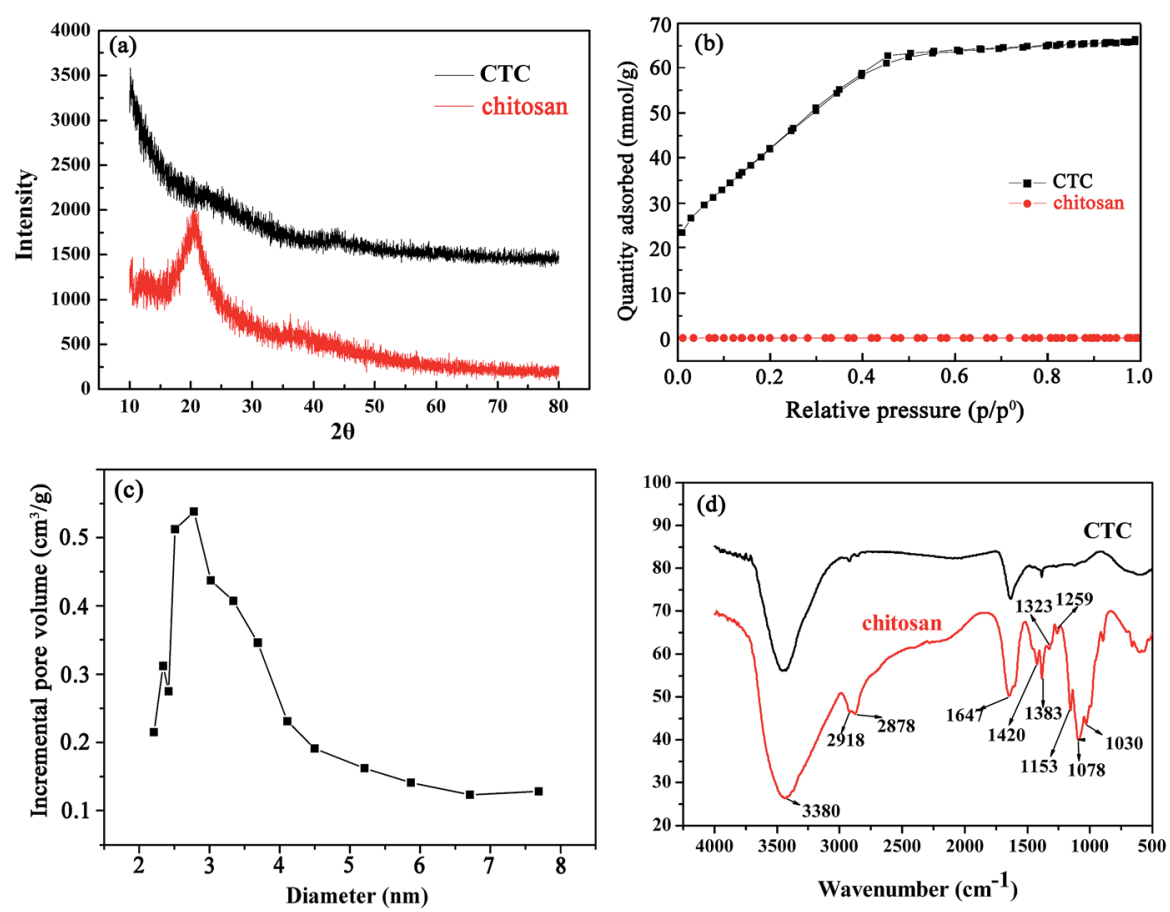

Fig. 1 (a) XRD patterns of chitosan and CTC; (b) nitrogen adsorption/desorption isotherms of chitosan and CTC; (c) pore size distributions of CTC obtained by the BJH method; (d) FTIR spectrum of chitosan and CTC. 
groups of the original chitosan were greatly altered by precarbonization and activation with $\mathrm{KOH}$ at various temperatures. The surface morphology of CTC is shown in Fig. 2, and provides information on the size and structures of CTC. Various pores formed on the surface of CTC after pre-carbonization and activation with $\mathrm{KOH}$ at different temperatures. The largediameter channels in CTC are presented in Fig. 2a, and countless small-diameter pores attached to the wall of large-diameter channels (Fig. 2b). Therefore, the obtained CTC has numerous pores that contribute to the remarkable surface area of CTC.

\subsection{Fast wastewater treatment experiments}

The performance during the fast treatment of wastewater is an important reference for the practical availability of adsorbents. ${ }^{50}$ In this study, another interesting finding is that CTC shows considerably fast wastewater treatment efficiency. We evaluated the removal efficiency of MB onto CTC using a homemade filter device (bottom area: $2.83 \times 10^{-3} \mathrm{~m}^{2}$, height: $0.1 \mathrm{~m}$ ), as shown in Fig. 3a. The flow rate of the aqueous solution is controlled at $1 \times 10^{3} \mathrm{~L} \mathrm{~m}^{-2} \mathrm{~h}^{-1}$ by a vacuum pump. The initial blue MB solution became clear after passing through the
CTC filter core (Fig. 3c). The effect of CTC dosage on the removal efficiency of MB from wastewater was investigated by adding MB solution $\left(10 \mathrm{mg} \mathrm{L}^{-1}\right)$ to the filter tube. A high removal rate of more than $93.4 \%$ was recorded for $4 \mathrm{~g}$ of absorbent (Fig. $4 \mathrm{a}$ ), and the wastewater treatment rate reached $250 \mathrm{~L} \mathrm{~g}^{-1} \mathrm{~h}^{-1}$. However, the removal rates significantly decreased when the volume of MB solution was over $150 \mathrm{~mL}$. As the speciation, degree of ionization, and surface charge of adsorbate are affected by solution $\mathrm{pH}^{49}$ the effect of $\mathrm{pH}$ on the removal efficiency of CTC was also determined with a certain amount of absorbent $(4 \mathrm{~g})$ and the volume of $\mathrm{MB}$ solution $(150 \mathrm{~mL}$, $100 \mathrm{mg} \mathrm{L}^{-1}$ ). The results are shown in Fig. 4b. The MB-CTC system was pH-dependent. The removal amount of MB slowly increased as the solution $\mathrm{pH}$ increased from 2 to 8 , followed by a significant increase from 8 to 12 . The maximum removal amount of $\mathrm{MB}$ was up to $163.67 \mathrm{mg} \mathrm{g}^{-1}$ at $\mathrm{pH} 10$. When the solution $\mathrm{pH}$ was in the range of 2 to 6 , less removal of $\mathrm{MB}$ from the solution may be due to the protonation of the amine groups on the CTC surface, resulting in the decrease in available adsorbent sites for dye cations. When the $\mathrm{pH}$ of solution continuously increased from 8 to 10 , the amount of protonated amine groups on the CTC surface might decrease, leading to the
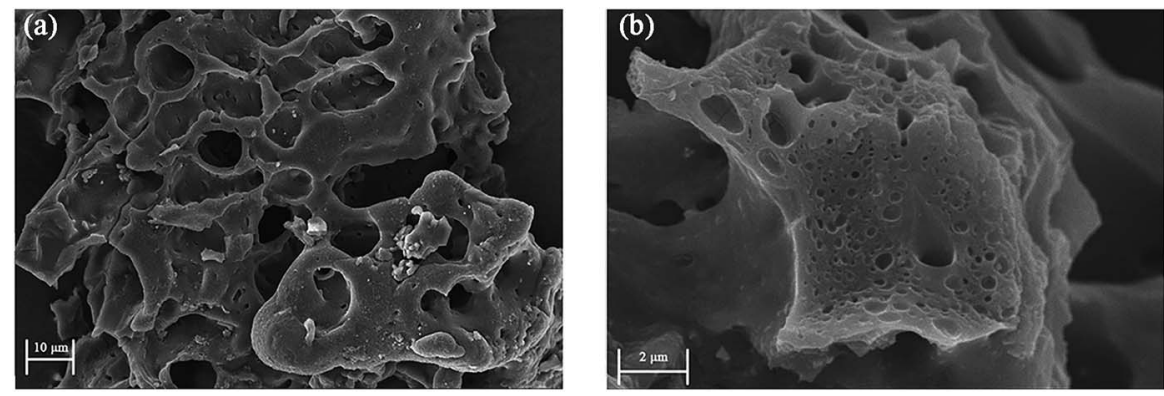

Fig. 2 SEM photographs of CTC. (a) Large-diameter channels through CTC. (b) Numerous pores distributed on CTC surface.

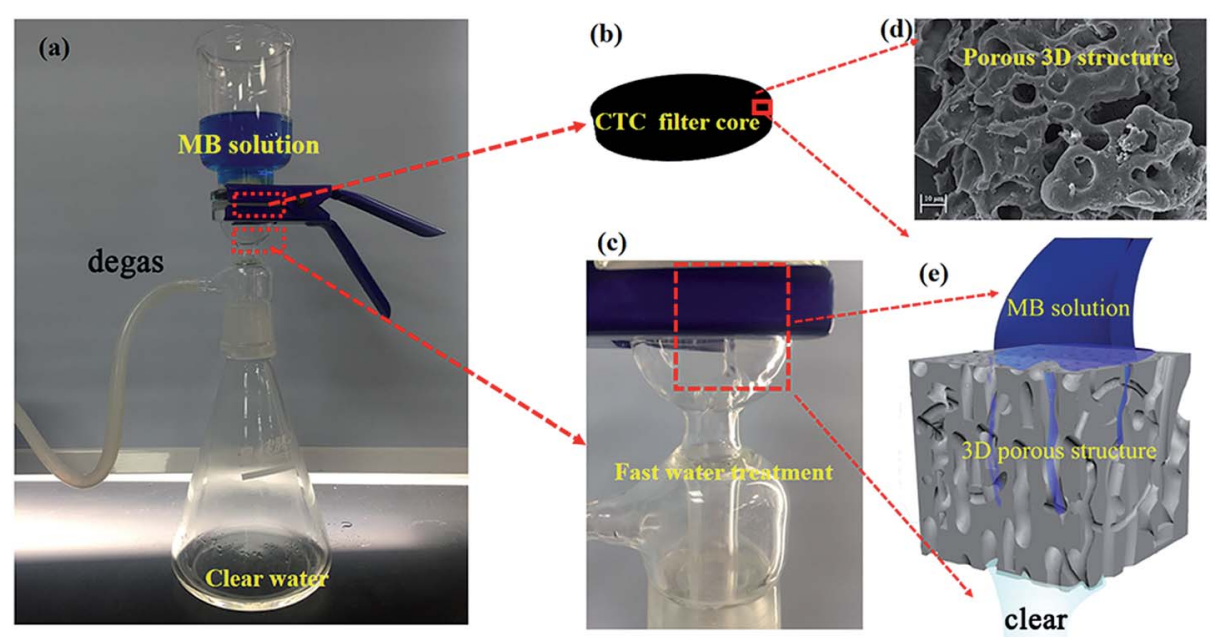

Fig. 3 (a) Experimental devices for fast water treatment experiments, where vacuum is applied to control water flow rates at $1 \times 10^{3} \mathrm{~L} \mathrm{~m}{ }^{-2} \mathrm{~h}^{-1}$. The blue solution in the funnel is MB solution $\left(10 \mathrm{mg} \mathrm{L}^{-1}\right.$ ); (b) CTC filter core with different doses; (c) close-up image of MB solution flowing through the CTC filter core. The initial blue MB solution changed to a colourless liquid after filtration. (d) Porous 3D microstructure of CTC. (e) Schematic of CTC for fast water treatment. 

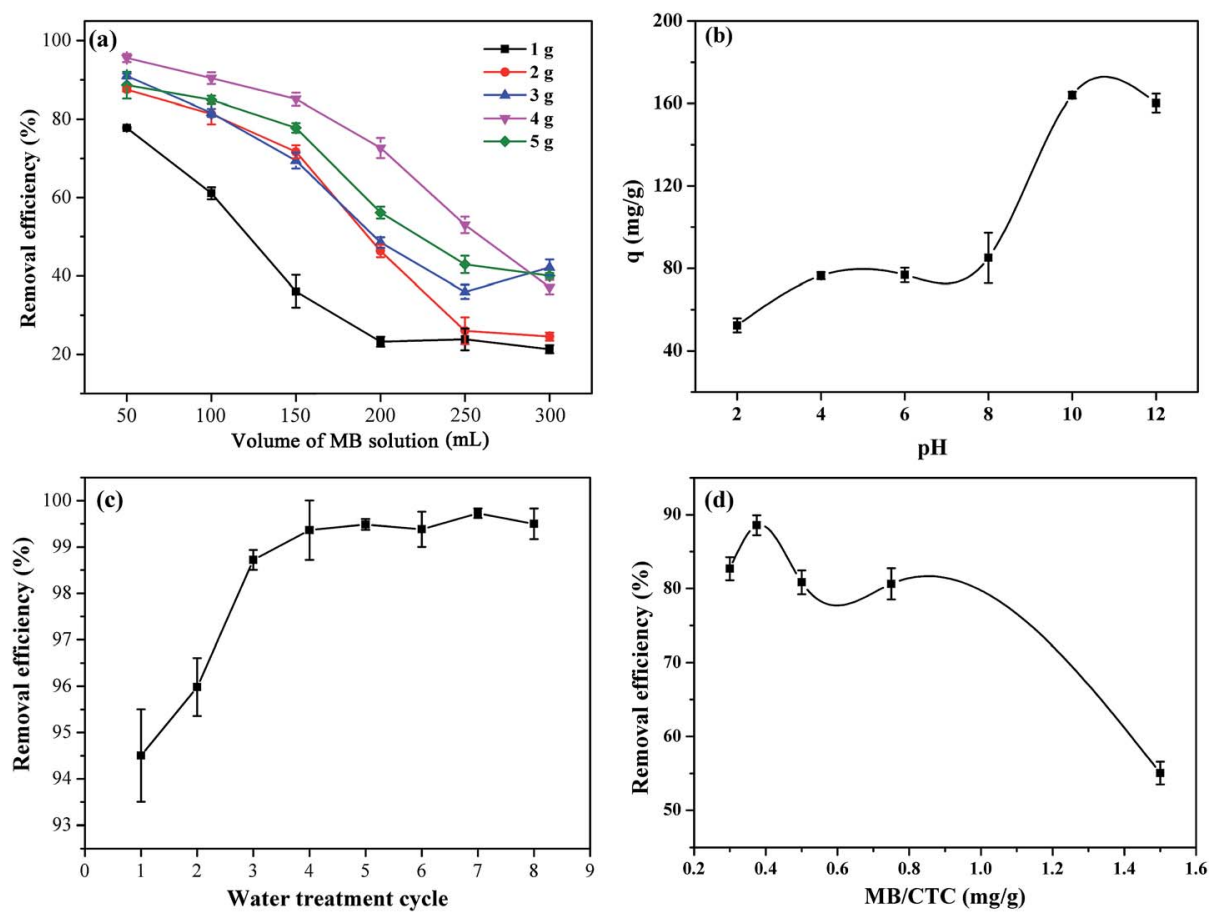

Fig. 4 (a) Removal efficiency of different amounts of MB on various doses of CTC ( $1 \mathrm{~g}, 2 \mathrm{~g}, 3 \mathrm{~g}, 4 \mathrm{~g}$, and $5 \mathrm{~g}$; MB concentration: $10 \mathrm{mg} \mathrm{L}^{-1}$ ). (b) Effect of solution $\mathrm{pH}$ on fast water treatment (CTC dose: $4 \mathrm{~g}, \mathrm{MB}$ concentration: $100 \mathrm{mg} \mathrm{L}^{-1}$ ). (c) Effect of number of water cycles on fast water treatment (CTC dose: $4 \mathrm{~g}$, MB concentration: $10 \mathrm{mg} \mathrm{L}^{-1}$ ). (d) Effect of ratios of MB amount in wastewater to weight of CTC (MB concentration: $10 \mathrm{mg} \mathrm{L}^{-1}$ ).

an obvious increase in the amount of MB from the solution. For the fast treatment of wastewater, the removal efficiency of $\mathrm{MB}$ on CTC was determined after various cycle times. The results showed that the adsorption efficiency was over $99.4 \%$ when the MB solution was cycled 4 times (Fig. 4c). To maximize the fast removal efficiency of CTC, we set a series of ratios of the amount of MB in wastewater to the weight of CTC. We found that fast removal efficiency was highest when the ratio was 0.375 (Fig. 4d). Therefore, we can determine the appropriate dosage of CTC according to the volume of wastewater and the concentration of dyes in wastewater to avoid waste.

The excellent fast wastewater treatment performance of the CTC filter element was due to the 3D porous structures of CTC. These numerous pores played different roles as wastewater passed through the material. The large channels in CTC were conducive to the rapid passage of water. The small-diameter pores were attached on the wall of channels, which increased the chances of interaction between $\mathrm{MB}$ in solution and the active groups in CTC as MB solution passed through the channels. The 3D porous structures consisting of largediameter channels and numerous pores enabled the rapid removal of MB from the flowing wastewater. Some studies have reported that materials with various $3 \mathrm{D}$ porous structures generally have excellent removal efficiency in fast wastewater treatment. ${ }^{42}$ The $3 \mathrm{D}$ porous structures of CTC loaded more active groups, which can be extended to other sewage treatments with special requirements. Therefore, CTC is not only an excellent adsorbent but also a promising base material for special adsorption.

\subsection{Adsorption properties of MB on CTC}

3.3.1 Effect of adsorbent dose. The effect of CTC dosage on the removal of MB from aqueous solutions was performed by dispersing different amounts (0.01-0.04 g) of CTC into a specific concentration of MB solution $\left(100 \mathrm{mg} \mathrm{L}^{-1}\right)$. The mixtures were stirred at $298 \mathrm{~K}$ and maintained for $8 \mathrm{~h}$. The effect of adsorbent dose on the removal of MB is shown in Fig. 5a. There was an obvious increase from $89.12 \%$ to $99.50 \%$ adsorption efficiency when the CTC dosage ranged from 0.01 to $0.04 \mathrm{~g}$. This increased adsorption could be explained by the increase in adsorbent sites available for dye cations, as induced by the increased CTC dosage. However, the adsorption capacities of MB onto CTC decreased from 956.23 to $249.43 \mathrm{mg} \mathrm{g}^{-1}$ when the CTC dose ranged from 0.01 to $0.04 \mathrm{~g}$. Therefore, the optimal dosage of CTC is $0.01 \mathrm{~g}$ because both the removal efficiency and adsorption capacity are relatively high.

3.3.2 Effect of ionic strength. The solution's ionic strength is another significant factor that affects dye adsorption. Fig. 5b shows the effect of ionic strength on the adsorption capacity of MB on CTC at different concentrations of NaCl. Different concentrations of $\mathrm{NaCl}$ ranged from 0 to $1 \mathrm{M}$ and were mixed with a specific concentration of MB solution $\left(100 \mathrm{mg} \mathrm{L}^{-1}, 100\right.$ $\mathrm{mL}$ ) with $0.01 \mathrm{~g}$ of adsorbent to determine the effect of salt (ionic strength) on the adsorption process. The samples were maintained for $8 \mathrm{~h}$ at $298 \mathrm{~K}$. As seen from Fig. 5b, there is a slight effect on the removal of $\mathrm{MB}$ with increasing $\mathrm{NaCl}$ concentration. This result was similar to those of previous reports. ${ }^{51,52}$ During the adsorption of MB on CTC, the available sites for MB molecules could be occupied by ions from the salt 

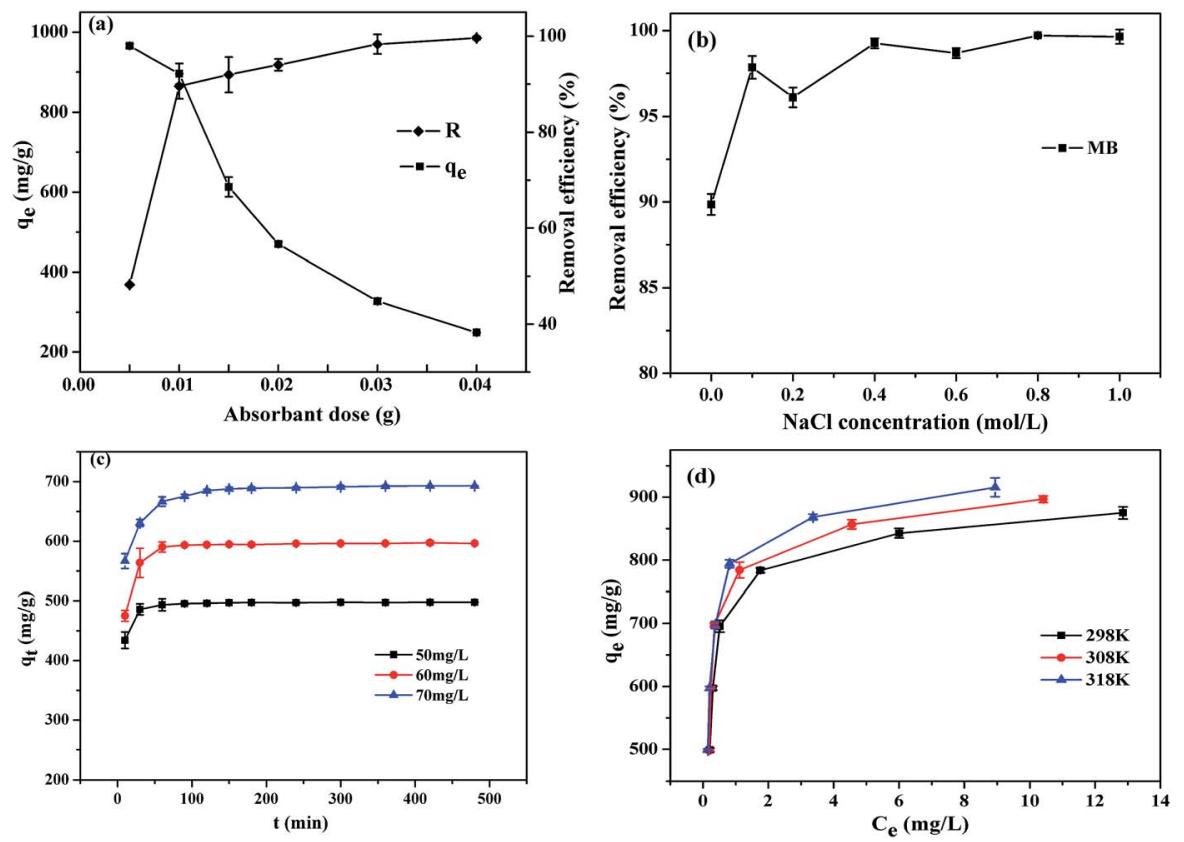

Fig. 5 (a) Effect of adsorbent dose on adsorption of MB (MB concentration: $100 \mathrm{mg} \mathrm{L}^{-1}$, temperature: $298 \mathrm{~K}$, contact time: 8 h); (b) effect of various concentrations of $\mathrm{NaCl}$ solution on percentage removal of $\mathrm{MB}$ (adsorbent dose: $0.01 \mathrm{~g}, \mathrm{MB}$ concentration: $100 \mathrm{mg} \mathrm{L}{ }^{-1}$, temperature: 298 $\mathrm{K}$, contact time: $8 \mathrm{~h}$ ); (c) effects of initial concentration and contact time on adsorption of MB onto CTC (adsorbent dose: $0.01 \mathrm{~g}$, temperature: $298 \mathrm{~K}$ ); (d) adsorption isotherms of MB onto CTC (adsorbent dose: $0.01 \mathrm{~g}$, contact time: $8 \mathrm{~h}$ ).

due to the competitive effect. However, even with a high concentration of $\mathrm{NaCl}\left(1.0 \mathrm{~mol} \mathrm{~L}^{-1}\right)$, there was a slight change in the removal capacity of $\mathrm{MB}$ on CTC, so CTC is an excellent adsorbent to remove MB in a high-salinity aqueous solution.

3.3.3 Effect of initial concentration and contact time. The adsorption experiments for MB on CTC were conducted at $298 \mathrm{~K}$ with different initial $\mathrm{MB}$ concentrations $\left(50,60,70 \mathrm{mg} \mathrm{L}^{-1}\right)$ and a CTC dosage of $0.01 \mathrm{~g}$. The reaction time intervals of the experiment were $8 \mathrm{~h}$, and the amount of absorbed MB was recorded. The results are shown in Fig. $5 \mathrm{c}$. In the first $30 \mathrm{~min}$, the adsorption rates rapidly increased. After 60, 90 and $120 \mathrm{~min}$, the reaction reached equilibrium in the reaction systems with initial concentrations of $\mathrm{MB}$ of 50,60 and $70 \mathrm{mg} \mathrm{L}^{-1}$. In the initial stage, there were many unoccupied adsorption sites for MB to adhere to, so the adsorption rate was high. However, the adsorption rate decreased when these adsorption sites were occupied by MB molecules. The lengthened time to reach equilibrium with the initial concentration of MB increased, mainly due to fiercer competition of MB molecules for active adsorption sites on CTC. The result is consistent with the report of Hameed et al. ${ }^{53}$ The amount of adsorbed MB onto CTC increased from 498.37 to $696.55 \mathrm{mg} \mathrm{g}^{-1}$ with the increasing initial concentration of $\mathrm{MB}$ from 50 to $70 \mathrm{mg} \mathrm{L}^{-1}$, mainly due to the increase in the driving force caused by the concentration gradient.

3.3.4 Effect of initial concentration and temperature. Three temperatures were established at $298 \mathrm{~K}, 308 \mathrm{~K}$ and $318 \mathrm{~K}$ in the experiment. The specific steps were to add $0.01 \mathrm{~g}$ CTC to MB solutions, with initial concentrations ranging from 50 to $100 \mathrm{mg} \mathrm{L}^{-1}$. The results were recorded after equilibrium for approximately $8 \mathrm{~h}$. The results are shown in Fig. $5 \mathrm{~d}$. The adsorption capability gradually increased with an increase in dye concentration and temperature. The adsorption capability of MB on CTC increased from 498.54 to $925.93 \mathrm{mg} \mathrm{g}^{-1}$ when the MB concentration increased from 50 to $100 \mathrm{mg} \mathrm{L}^{-1}$ at $318 \mathrm{~K}$. The results indicated that the initial MB concentration is one factor that influences dye removal. This may be due to the necessary driving force provided by the initial dye concentration, which offset MB mass transfer resistance between the solid and aqueous phases. According to previous reports, temperature is another key factor for the adsorption process. The adsorption capacity of $\mathrm{MB}$ (initial concentration $100 \mathrm{mg} \mathrm{g}^{-1}$ ) on CTC increases from $877.19 \mathrm{mg} \mathrm{g}^{-1}$ at $298 \mathrm{~K}$ to $925.93 \mathrm{mg} \mathrm{g}^{-1}$ at $318 \mathrm{~K}$. MB molecules have a greater chance to cross the external boundary layer and the internal pores of the CTC particle when the temperature is improved, and thus diffusion more easily occurs. Adsorption is an endothermic reaction according to the result, in which the adsorption capacity increases with increasing temperature. Meanwhile, the maximum adsorption capacity of MB on CTC was $925.93 \mathrm{mg} \mathrm{g}^{-1}$ at $318 \mathrm{~K}$, which is remarkable compared to some reported absorbents (Table 1). The removal of MB by adsorption onto CTC was due to MB in the $\mathrm{MB}^{+}$cationic form. The mechanism for the removal of dye by adsorption may involve the following steps. The first step is the migration of dye from wastewater to the surface of the adsorbent (CTC). The second step is the diffusion of dye through the boundary layer to the surface of the adsorbent. Third, the dye combines with active sites on the surface of the adsorbent and diffuses into the interior pores of the adsorbent particle. In addition, the huge surface area of CTC provides more active sites and ensures efficient dye diffusion into the interior pores of the CTC particle. CTC is thus promising for application in wastewater treatment. 
Table 1 Comparison of adsorption of MB onto various adsorbents

\begin{tabular}{|c|c|c|c|}
\hline Adsorbents & $\begin{array}{l}T \\
\left({ }^{\circ} \mathrm{C}\right)\end{array}$ & $\begin{array}{l}\text { Maximum } \\
\text { adsorption } \\
\text { capacities } \\
\left(\mathrm{mg} \mathrm{g}^{-1}\right)\end{array}$ & References \\
\hline Biomass-based activated carbon & 30 & 259.25 & 54 \\
\hline Sludge-based activated carbon & 30 & 263.16 & 55 \\
\hline Bamboo-based activated carbon & 30 & 454.2 & 56 \\
\hline Activated carbons from walnut shells & 25 & 315 & 57 \\
\hline $\begin{array}{l}\text { Chitosan-derived three- } \\
\text { dimensional carbon }\end{array}$ & 25 & 890.32 & This study \\
\hline
\end{tabular}

The equilibrium data indicate the detailed characteristics of the adsorption capacity or dosage of CTC for removing pollutant under the given system conditions, namely, adsorption isotherms, which provide important bases to design adsorption systems. ${ }^{58-60}$ In the present study, we analysed the equilibrium data to determine the Langmuir or Freundlich isotherm model equations to fit the equilibrium data.

The Langmuir model assumes the adsorption of a single adsorbate onto a series of equivalent sites on the surface of the solid, with equal affinity and energy among binding sites. The adsorbed species have no interaction. The Langmuir isotherm model can be expressed by the following eqn (4):

$$
\frac{C_{\mathrm{e}}}{q_{\mathrm{e}}}=\frac{1}{q_{\max } \times K_{\mathrm{L}}}+\frac{C_{\mathrm{e}}}{q_{\max }}
$$

where $C_{\mathrm{e}}\left(\mathrm{mg} \mathrm{L}^{-1}\right)$ and $q_{\mathrm{e}}\left(\mathrm{mg} \mathrm{g}^{-1}\right)$ are the dye concentration and the amount of adsorbed MB at equilibrium, respectively. $K_{\mathrm{L}}$ $\left(\mathrm{L} \mathrm{mg}^{-1}\right)$ and $q_{\max }\left(\mathrm{mg} \mathrm{g}^{-1}\right)$ are the Langmuir constant and the maximum adsorption capacity, respectively. The Langmuir constant is related to the binding energy and the monolayer capacity of the sorption system. $R_{\mathrm{L}}$ is an equilibrium constant to determine whether the adsorption process is favourable, and $R_{\mathrm{L}}$ is calculated by eqn (5):

$$
R_{\mathrm{L}}=\frac{1}{1+K_{\mathrm{L}} \times C_{0}}
$$

where $K_{\mathrm{L}}\left(\mathrm{L} \mathrm{mg}^{-1}\right)$ and $C_{0}\left(\mathrm{mg} \mathrm{L}^{-1}\right)$ are the Langmuir constant and initial MB concentration, respectively.

The adsorption isotherms are unfavourable, linear, and favourable when the $R_{\mathrm{L}}$ values are $>1,=1$, and $<1$, respectively. According to all values of $R_{\mathrm{L}}$ calculated by the above equation, the range of $R_{\mathrm{L}}$ is between 0 and 1, which indicated that the adsorption process of MB on CTC is favourable.
The Freundlich isotherm assumes that the adsorption of dyes occurs on heterogeneous surface by multilayer adsorption, which is sometimes non-ideal. It also assumes that the amount of adsorbed dye increases immensely when the concentration of initial dye increases. The Freundlich isotherm model equation is as follows:

$$
\log q_{\mathrm{e}}=\log K_{\mathrm{F}}+\frac{\log C_{\mathrm{e}}}{n}
$$

The Langmuir and Freundlich isotherm model constants, along with the parameters and correlation coefficients, are summarized in Table 2. According to the correlation coefficient values $\left(R^{2}\right)$, the Langmuir model better describes the process of adsorption. The maximum adsorption capacity of CTC was $925.93 \mathrm{mg} \mathrm{g}^{-1}$ at $318 \mathrm{~K}$, and the physical monolayer adsorption of MB on CTC occurs at specific homogeneous sites.

\subsection{Kinetic studies}

Kinetic models are usually used to explore the controlling mechanisms between dyes and the absorbent. Our results are shown in Fig. 6. The initial adsorption capacity of MB on CTC was high and sharply increased within $30 \mathrm{~min}$. Thus, it is important to know the concrete controlling mechanisms for the adsorption process of $\mathrm{MB}$ on CTC. There were numerous chemical groups on the pore surface of CTC, which implied more types of interactions between MB and CTC. The kinetic models include pseudo-first-order, pseudo-second-order and intra-particle diffusion. ${ }^{44,45,56}$ Through these kinetic models, the behaviour of MB onto CTC was better understood. $R^{2}$ represents the correlation coefficient of the model, which was used to judge whether the studied equation was suitable for the adsorption process. First, we used the pseudo-first-order equation to fit the experimental data as follows:

$$
\frac{\mathrm{d} q_{t}}{\mathrm{~d} t}=k_{1} \times\left(q_{\mathrm{e}}-q_{t}\right)
$$

We applied the boundary conditions $\left(q_{t}=0\right.$ at $t=0$ and $q_{t}=$ $q_{t}$ at $t=t$ ) for definite integration, and eqn (7) shifted as follows:

$$
\log \left(q_{\mathrm{e}}-q_{t}\right)=\log q_{\mathrm{e}}-\frac{k_{1} \times t}{2.303}
$$

where $q_{\mathrm{e}}$ and $q_{t}$ represent the amount of MB absorbed at equilibrium and a specific time $(t)$, respectively, and $k_{1}$ represents the pseudo-first-order equation rate constant. The values of $\log \left(q_{\mathrm{e}}-q_{t}\right)$ versus $t$ were obtained from the experimental kinetic

Table 2 Isotherm constants for adsorption of MB

\begin{tabular}{lllllll}
\hline & \multicolumn{3}{l}{ Langmuir model } & & & \multicolumn{2}{c}{ Freundlich model } \\
\cline { 2 - 4 }$T(\mathrm{~K})$ & $q_{\text {max }}\left(\mathrm{mg} \mathrm{g}^{-1}\right)$ & $K_{\mathrm{L}}\left(\mathrm{L} \mathrm{mg}^{-1}\right)$ & $R^{2}$ & & $K_{\mathrm{F}}\left(\mathrm{L} \mathrm{g}^{-1}\right)$ & $1 / n$ \\
298 & 890.32 & 5.6542 & 0.99986 & 680.72 & 0.11936 \\
308 & 909.09 & 6.4663 & 0.99978 & 709.50 & 0.12362 \\
318 & 925.93 & 7.2561 & 0.99988 & 732.11 & 0.85468 \\
& & & & & 0.13114
\end{tabular}



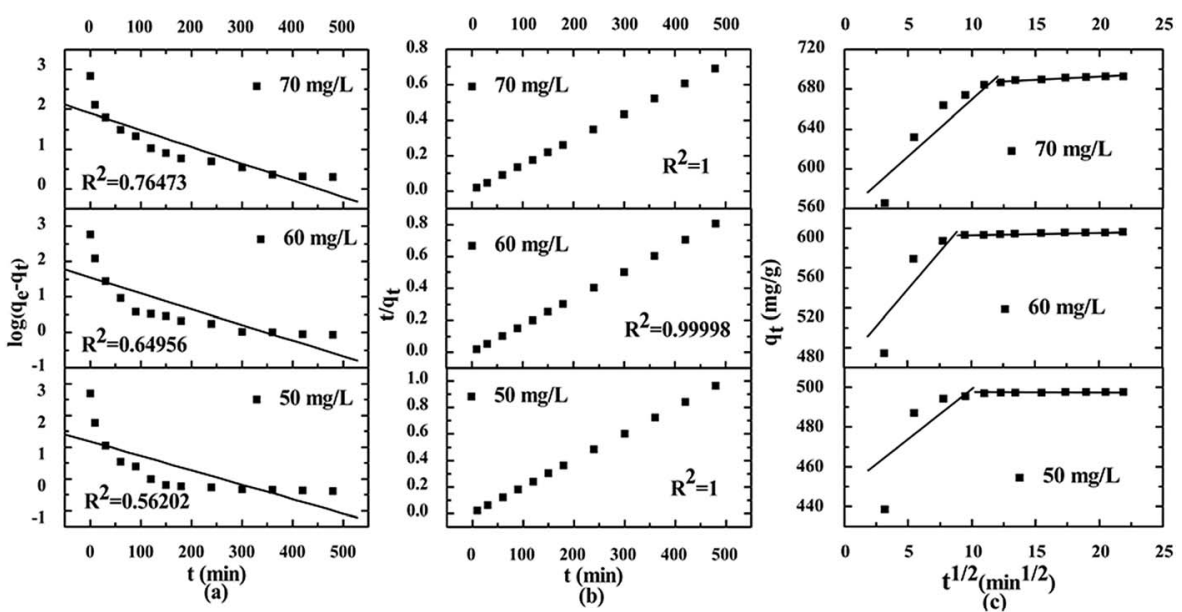

Fig. 6 Adsorption kinetics for MB adsorption by CTC (adsorbent dose: 0.01 g, temperature: 298 K, contact time: 8 h). (a) Pseudo-first-order; (b) pseudo-second-order; (c) intra-particle diffusion model.

data at various concentrations of $\mathrm{MB}$, and the results are shown in Fig. 6a. The pseudo-first-order constants obtained from experiments are presented in Table 3. All calculated correlation coefficients $R^{2}$ were low, ranging from 0.39171 to 0.64956 . In addition, the calculated theoretic values of $q_{\mathrm{e}, \mathrm{cal}}$ are far from the experimental values of $q_{\mathrm{e} \text {,exp }}$. According to the above results, we suggest that the pseudo-first-order model is not suitable to describe the adsorption process of MB on CTC.

Another model of kinetic models, pseudo-second-order, could describe the adsorption process, presented as the following equation:

$$
\frac{\mathrm{d} q_{t}}{\mathrm{~d} t}=k_{2} \times\left(q_{\mathrm{e}}-q_{t}\right)^{2}
$$

where $k_{2}$ is the pseudo-second-order rate constant. Under the boundary conditions $q_{t}=0$ at $t=0$ and $q_{t}=q_{t}$ at $t=t$, we integrated eqn (9) and obtained the simple and linear equation as follows:

Table 3 Kinetic parameters for MB adsorption onto CTC

\begin{tabular}{llll}
\hline & \multicolumn{2}{l}{ Dye concentration $\left(\mathrm{mg} \mathrm{L}^{-1}\right)$} & \\
\cline { 2 - 3 } Kinetic models & 50 & 60 & 70 \\
\hline$q_{\mathrm{e}, \text { exp }}\left(\mathrm{mg} \mathrm{g}^{-1}\right)$ & 497.95 & 597.03 & 694.85 \\
Pseudo-first-order & & & \\
$q_{\mathrm{e}, \text { cal }}\left(\mathrm{mg} \mathrm{g}^{-1}\right)$ & 15.07 & 35.69 & 80.30 \\
$K_{1}\left(\mathrm{~min}^{-1}\right)$ & 0.01040 & 0.01030 & 0.00972 \\
$R_{1}{ }^{2}$ & 0.56202 & 0.64956 & 0.76473 \\
Pseudo-second-order & & & \\
$q_{\mathrm{e}, \text { cal }}\left(\mathrm{mg} \mathrm{g}^{-1}\right)$ & 498.51 & 598.80 & 699.30 \\
$K_{2}\left(\mathrm{~g} \mathrm{mg}^{-1} \mathrm{~min}^{-1}\right)$ & 0.00406 & 0.00127 & 0.00053 \\
$R_{2}{ }^{2}$ & 1 & 0.99998 & 1 \\
Intra-particle diffusion & & & \\
$k_{\mathrm{P}}\left(\mathrm{mg} \mathrm{g}^{-1} \mathrm{~min}^{-1 / 2}\right)$ & 1.74 & 3.71 & 4.94 \\
$R_{3}{ }^{2}$ & 0.70807 & 0.87092 & 0.84620
\end{tabular}

$$
\frac{t}{q_{t}}=\frac{q_{\mathrm{e}}{ }^{2}}{k_{2}}+\frac{t}{q_{\mathrm{e}}}
$$

The values of $q_{\mathrm{e}}$ and $k_{2}$ are obtained experimentally from the slope and the intercept of plot $t / q_{t}$ versus $t$. The linear plot of $t / q_{t}$ versus $t$ is shown in Fig. 6b, which facilitates the applicability of the pseudo-second-order equation. The detailed kinetic results are shown in Table 3 , including $k_{2}, q_{\mathrm{e}}\left(q_{\mathrm{e} \text {,exp }}\right)$ and $R_{2}{ }^{2}$. According to the data in Table 3 , the adsorption processes are well explained by the pseudo-second-order model because the correlation coefficients $R_{2}{ }^{2}$ are higher than 0.9999 .

Finally, we used the intra-particle diffusion model to fit the experimental data. It is possible that there is intraparticle diffusion during adsorption, and the intra-particle diffusion equation is as follows:

$$
q_{t}=k_{\mathrm{p}} \times t^{1 / 2}+I
$$

where $k_{\mathrm{p}}$ is the intra-particle diffusion rate constant. The equation plots of $q_{t}$ versus $t^{1 / 2}$ of $\mathrm{MB}$ at different initial concentrations are shown in Fig. $6 \mathrm{c}$. The rate constants $k_{\mathrm{p}}, I, R_{3}{ }^{2}$ are listed in Table 3 . The correlation coefficients $R_{3}{ }^{2}$ are between 0.70807 and 0.87092 , lower than the correlation coefficients $R_{2}{ }^{2}$. Hence, the intra-particle diffusion model is not suitable to describe the adsorption of MB on CTC.

\subsection{Thermodynamic parameters}

The adsorption study was performed at 298, 308 and $318 \mathrm{~K}$. The adsorption thermodynamic parameters were calculated according to the following equations, including changes in free energy $\left(\Delta G^{0}\right)$, enthalpy $\left(\Delta H^{0}\right)$, and entropy $\left(\Delta S^{0}\right)$.

$$
\Delta G^{0}=-R_{\text {gas }} \times T \times \operatorname{In} K_{\mathrm{L}}
$$

where, at equilibrium for the above equation, $K_{\mathrm{L}}\left(\mathrm{L} \mathrm{mg}^{-1}\right)$ represents the Langmuir constant, and $R_{\text {gas }}\left(8.314 \mathrm{~J} \mathrm{~mol}^{-1} \mathrm{~K}^{-1}\right)$ and $T(\mathrm{~K})$ represent the universal gas constant and the solution 
temperature, respectively. $\Delta G^{0}$ is the change in Gibbs free energy ( $\left.\mathrm{kJ} \mathrm{mol}{ }^{-1}\right)$, and $\Delta H^{0}\left(\mathrm{~kJ} \mathrm{~mol}^{-1}\right)$ and $\Delta S^{0}\left(\mathrm{~J}\left(\mathrm{~K}^{-1} \mathrm{~mol}^{-1}\right)\right)$ are the heat of reaction and the change in entropy, respectively. $\Delta G^{0}, \Delta H^{0}$ and $\Delta S^{0}$ are shown in Table 4. These results suggest that the adsorption of MB on CTC is spontaneous; $\Delta G^{0}$ is -4.29 at $298 \mathrm{~K},-4.78$ at $308 \mathrm{~K}$ and -5.24 at $318 \mathrm{~K}$. According to the above results, $\Delta G^{0}$ value is decreasing when the temperature slowly rises, which indicates that the adsorption process of MB on CTC is more active at higher temperature. The positive value of $\Delta H^{0}\left(9.86 \mathrm{~kJ} \mathrm{~mol}^{-1}\right)$ indicates that the adsorption process of MB on CTC is endothermic. The positive entropy change $\Delta S^{0}$ $\left(0.048 \mathrm{~kJ} \mathrm{~mol}^{-1} \mathrm{~K}^{-1}\right)$ shows that disorder occurs at the solidsolution interface during $\mathrm{MB}$ adsorption. As the temperature increases, the decrease in the mobility of MB molecules enables the molecules to more easily adsorb onto the solid phase.

\subsection{Desorption and reusability}

The desorption efficiency and regeneration potential of absorbents is critical for the absorbent's economy and feasibility. Therefore, we studied the desorption efficiency and regeneration potential of CTC by the batch method using various desorption agents, such as distilled water, $0.1 \mathrm{M}$ of EDTA, $0.1 \mathrm{M}$ of hydrochloric acid, ethyl alcohol, and $0.1 \mathrm{M}$ of oxalic acid solutions. The adsorption efficiencies of MB on CTC were summarized after repeated adsorption-desorption by five desorption agents (Fig. 7). The two best desorption agents are listed in Fig. 8. The results indicated that the highest desorption effect for $\mathrm{MB}$ was obtained using ethyl alcohol, and the adsorption efficiency of MB on CTC was not significantly decreased after 6 cycles; the efficiency was above 93\%. Oxalic acid showed the second highest desorption effect. After 6 adsorption-desorption cycles of MB on CTC using $0.1 \mathrm{M}$ oxalic

Table 4 Thermodynamic parameters for MB adsorption onto CTC

\begin{tabular}{llllll}
\hline & & & & $\Delta G^{0}\left(\mathrm{~kJ} \mathrm{~mol}^{-1}\right)$ & \\
\cline { 4 - 6 } Absorbent & $\begin{array}{l}\Delta H^{0} \\
\left(\mathrm{~kJ} \mathrm{~mol}^{-1}\right)\end{array}$ & $\begin{array}{l}\Delta S^{0} \\
\left(\mathrm{~kJ} \mathrm{~mol}^{-1} \mathrm{~K}^{-1}\right)\end{array}$ & $298 \mathrm{~K}$ & $308 \mathrm{~K}$ & $318 \mathrm{~K}$ \\
\hline CTC & 9.86 & 0.048 & -4.29 & -4.78 & -5.24
\end{tabular}

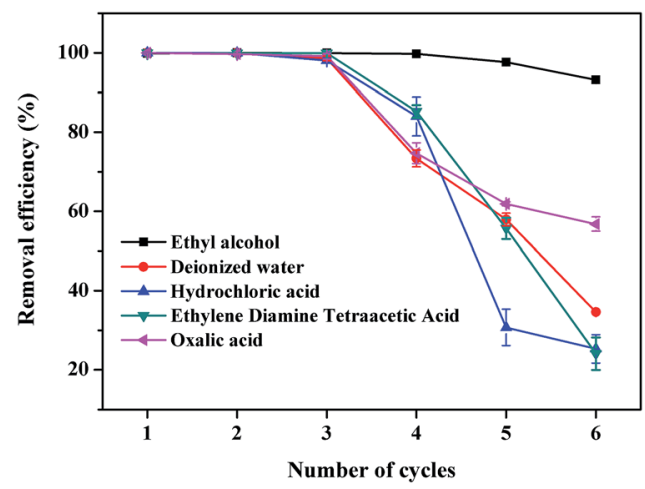

Fig. 7 Effect of adsorption-desorption cycles on removal efficiency of $\mathrm{MB}$ on CTC.

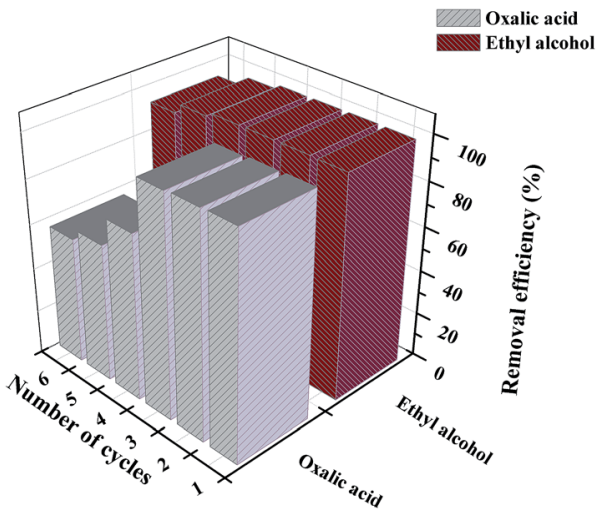

Fig. 8 Removal efficiency of CTC after repeated adsorptiondesorption operations.

acid solutions, the removal efficiency of MB on CTC decreased to $55 \%$. Incomplete desorption can be attributed to the physical adsorption between adsorbent and MB. ${ }^{61}$ From the above results, CTC was a good reusable adsorbent and can be successfully applied to the removal of dyes from wastewater.

\section{Conclusions}

In summary, we prepared CTC with 3D porous structures consisting of mesopores and large-diameter channels via precarbonization and activation with $\mathrm{KOH}$. The obtained CTC is applied as a low-cost and environmental friendly adsorbent for the removal of basic dyes. The results indicated that CTC has a remarkable adsorption capacity towards MB up to $925.83 \mathrm{mg}$ $\mathrm{g}^{-1}$ in the equilibrium condition. Meanwhile, the excellent fast removal capability of $\mathrm{MB}$ from flowing wastewater was obtained, with a high removal rate up to $93.4 \%$ when the wastewater flowing rate was $250 \mathrm{~L} \mathrm{~g}^{-1} \mathrm{~h}^{-1}$. These excellent results are primarily due to the increased accessibility of active sites between MB molecules and CTC and the rich suitable pores for the diffusion of MB molecules. In addition, CTC was a good reusable adsorbent and could be successfully applied to recover MB from wastewater. Therefore, the obtained CTC could be a promising absorbent that can be widely used in practice. Our findings will encourage more efforts to design 3D porous structures in natural materials using controllable activation methods, and the obtained materials can be utilized as base materials for further modification.

\section{Conflicts of interest}

There are no conflicts to declare.

\section{Acknowledgements}

This work was supported by National Natural Science Foundation of China (Grant No. 41401564) and International cooperation project of Ministry of Science and technology (2015DFA11660). 


\section{References}

1 J. Sokolowska-Gajda, H. S. Freeman and A. Reife, Dyes Pigm., 1996, 30, 1-20.

2 I. Kabdaşli, O. Tünay and D. Orhon, Water Sci. Technol., 1999, 40, 261-267.

3 J. F. Gao, Q. Zhang, J. H. Wang, X. L. Wu, S. Y. Wang and Y. Z. Peng, Bioresour. Technol., 2011, 102, 805.

4 Y. C. Wong, Y. S. Szeto, W. H. Cheung and G. McKay, Langmuir, 2003, 19, 7888-7894.

5 Y. Gao, C. Li, J. Shen, H. Yin, X. An and H. Jin, J. Food Sci., 2011, 76, 125-129.

6 Y. C. Wong, Y. S. Szeto, W. H. Cheung and G. Mckay, Process Biochem., 2004, 39, 695-704.

7 N. Azbar, T. Yonar and K. Kestioglu, Chemosphere, 2004, 55, 35-43.

8 J. W. Lee, S. P. Choi, R. Thiruvenkatachari, W. G. Shim and H. Moon, Dyes Pigm., 2006, 69, 196-203.

9 J. H. Mo, H. L. Yong, J. Kim, J. Y. Jeong and J. Jegal, Dyes Pigm., 2008, 76, 429-434.

10 P. Sharma, H. Kaur, M. Sharma and V. Sahore, Environ. Monit. Assess., 2011, 183, 151.

11 O. H. Duygu and J. H. Van Leeuwen, J. Food Eng., 2010, 101, 106-112.

12 J. S. Piccin, C. S. Gomes, L. A. Feris and M. Gutterres, Chem. Eng. J., 2012, 183, 30-38.

13 A. Tabara, C. Yamane, M. Abe and M. Seguchi, Cellulose, 2011, 18, 45-55.

14 K. Siwińska-Stefańska, M. Nowacka and T. Jesionowski, Dyes Pigm., 2012, 94, 338-348.

15 L. Yu, W. W. Li, M. H. Lam and H. Q. Yu, Appl. Microbiol. Biotechnol., 2011, 90, 1119.

16 A. Debrassi, A. F. Corrêa, T. Baccarin, N. Nedelko, A. ŚlawskaWaniewska, K. Sobczak, P. Dłużewski, J. M. Greneche and C. A. Rodrigues, Chem. Eng. J., 2012, 183, 284-293.

17 G. Crini, Bioresour. Technol., 2006, 97, 1061-1085.

18 L. Huo, S. Zhang, X. Guo, F. Xu, Y. Li and J. Hou, Angew. Chem., 2011, 50, 9697.

19 R. A. Muzzarelli, Chitin, Elsevier, 2013.

20 M. S. Chiou and H. Y. Li, Chemosphere, 2003, 50, 1095.

21 S. Chatterjee, S. Chatterjee, B. P. Chatterjee, A. R. Das and A. K. Guha, J. Colloid Interface Sci., 2005, 288, 30.

22 G. Annadurai, L. Y. Ling and J. F. Lee, J. Hazard. Mater., 2008, $152,337$.

23 Z. Bekçi, C. Özveri, Y. Seki and K. Yurdakoç, J. Hazard. Mater., 2008, 154, 254-261.

24 G. Crini, F. Gimbert, C. Robert, B. Martel, O. Adam, N. Morincrini, G. F. De and P. M. Badot, J. Hazard. Mater., 2008, 153, 96-106.

25 M. Sarkar and P. Majumdar, Chem. Eng. J., 2011, 175, 376-387.

26 M. N. Kumar, R. A. Muzzarelli, C. Muzzarelli, H. Sashiwa and A. J. Domb, Chem. Rev., 2004, 104, 6017-6084.

27 L. Zhou, Y. Wang, Z. Liu and Q. Huang, J. Hazard. Mater., 2009, 161, 995.

28 A. Mohseni-Bandpi, B. Kakavandi, R. R. Kalantary, A. Azari and A. Keramati, RSC Adv., 2015, 5, 73279-73289.

29 A. H. Gedam and R. S. Dongre, RSC Adv. , 2016, 6., 22639-22652.
30 X. Li, Y. Li and Z. Ye, Chem. Eng. J., 2011, 178, 60-68.

31 G. Z. Kyzas and N. K. Lazaridis, J. Colloid Interface Sci., 2009, 331, 32.

32 R. P. Han, J. J. Zhang, P. Han, Y. F. Wang, Z. H. Zhao and M. S. Tang, Chem. Eng. J., 2009, 145, 496-504.

33 S. Sengupta, A. Goswami and R. Mondal, New J. Chem., 2014, 38, 2470-2479.

34 Z. Liu, H. Nie, Z. Yang, J. Zhang, Z. Jin, Y. Lu, Z. Xiao and S. Huang, Nanoscale, 2013, 5, 3283-3288.

35 X. Zhang, D. Liu, L. Yang, L. Zhou and T. You, J. Mater. Chem. A, 2015, 3, 10031-10037.

36 F. L. Meng, Z. L. Wang, H. X. Zhong, J. Wang, J. M. Yan and X. B. Zhang, Adv. Mater., 2016, 28, 7948.

37 B. H. Hameed, A. A. Ahmad and N. Aziz, Chem. Eng. J., 2007, 133, 195-203.

38 Y. Xue, H. Hou and S. Zhu, Chem. Eng. J., 2009, 147, 272-279. 39 M. Sekar, V. Sakthi and S. Rengaraj, J. Colloid Interface Sci., 2004, 279, 307-313.

40 S. Senthilkumaar, P. R. Varadarajan, K. Porkodi and C. V. Subbhuraam, J. Colloid Interface Sci., 2005, 284, 78-82. 41 Y. Liu, Y. Tian, C. Luo, G. Cui and S. Yan, New J. Chem., 2015, 39, 5484-5492.

42 F. Chen, A. S. Gong, M. Zhu, G. Chen, S. D. Lacey, J. Feng, Y. Li, Y. Wang, J. Dai and Y. Yao, ACS Nano, 2017, 11, 4275. 43 S. Kumar and J. Koh, Int. J. Mol. Sci., 2012, 13, 6102-6116.

44 C. Sheindorf, M. Rebhun and M. Sheintuch, J. Colloid Interface Sci., 1981, 79, 136-142.

45 M. A. Lillo-Ródenas, D. Cazorla-Amorós and A. LinaresSolano, Carbon, 2003, 41, 267-275.

46 L. Chunlan, X. Shaoping, G. Yixiong, L. Shuqin and L. Changhou, Carbon, 2005, 43, 2295-2301.

47 W. Qiao, A. Seongho Yoon and I. Mochida, Energy Fuels, 2006, 20, 1680-1684.

48 K. R. Hall, L. C. Eagleton, A. Acrivos and T. Vermeulen, Ind. Eng. Chem. Fundam., 1966, 5.

49 V. S. Mane, I. D. Mall and V. C. Srivastava, Dyes Pigm., 2007, 73, 269-278.

50 B. Farizoglu, A. Nuhoglu, E. Yildiz and B. Keskinler, Filtr. Sep., 2003, 40, 41-47.

51 Y. S. Al-Degs, M. I. El-Barghouthi, A. H. El-Sheikh and G. M. Walker, Dyes Pigm., 2008, 77, 16-23.

52 L. Wang, J. Zhang, R. Zhao, C. Li, Y. Li and C. L. Zhang, Desalination, 2010, 254, 68-74.

53 B. Hameed, A. Ahmad and N. Aziz, Chem. Eng. J., 2007, 133, 195-203.

54 S. K. Theydan and M. J. Ahmed, J. Anal. Appl. Pyrolysis, 2012, 97, 116-122.

55 W.-H. Li, Q.-Y. Yue, B.-Y. Gao, Z.-H. Ma, Y.-J. Li and H.-X. Zhao, Chem. Eng. J., 2011, 171, 320-327.

56 B. Hameed, A. M. Din and A. Ahmad, J. Hazard. Mater., 2007, 141, 819-825.

57 J. Yang and K. Qiu, Chem. Eng. J., 2010, 165, 209-217.

58 Y. S. Ho and G. Mckay, Process Biochem., 1999, 34, 451-465.

59 Y. S. Ho and C. C. Chiang, Adsorption, 2001, 7, 139-147.

60 H. Yuh-Shan, Scientometrics, 2004, 59, 171-177.

61 X. Li, Y. Li, S. Zhang and Z. Ye, Chem. Eng. J., 2012, 183, 88-97. 